\title{
Article \\ Optimized Cooling Power of a Vuilleumier Refrigerator with Limited Regeneration
}

\author{
Abdellah Khodja , Raphael Paul, Andreas Fischer and Karl Heinz Hoffmann * \\ Institut für Physik, Technische Universität Chemnitz, 09107 Chemnitz, Germany; \\ akhodja@uni-osnabrueck.de (A.K.); raphael.paul@physik.tu-chemnitz.de (R.P.); \\ andreas.fischer@physik.tu-chemnitz.de (A.F.) \\ * Correspondence: hoffmann@physik.tu-chemnitz.de
}

Citation: Khodja, A.; Paul, R.; Fischer, A.; Hoffmann, K.H. Optimized Cooling Power of a Vuilleumier Refrigerator with Limited Regeneration. Energies 2021, 14, 8376. https://doi.org/10.3390/en14248376

Academic Editor: Paweł Ocłoń

Received: 4 November 2021

Accepted: 2 December 2021

Published: 12 December 2021

Publisher's Note: MDPI stays neutral with regard to jurisdictional claims in published maps and institutional affiliations.

Copyright: (c) 2021 by the authors. Licensee MDPI, Basel, Switzerland. This article is an open access article distributed under the terms and conditions of the Creative Commons Attribution (CC BY) license (https:// creativecommons.org/licenses/by/ $4.0 /)$.

\begin{abstract}
Vuilleumier refrigerators provide cooling power by utilizing a heat source at temperatures above the ambient. This is particularly helpful in situations where waste heat is available and other power sources are limited. Vuilleumier refrigerators come in different technical configurations; here we analyze the thermodynamic performance of a configuration utilizing two displacer pistons with integrated regenerators. More specifically, we optimize the cooling power by optimizing the piston movement for a range of operation speeds. The optimization is based on the AS motion class for cyclic dynamics and uses an endoreversible model for the refrigerator. Our focus is on the influence of the regeneration extent present, and we find performance gains of about $17 \%$ for high regeneration extent and of about $28 \%$ for lower regeneration extent.
\end{abstract}

Keywords: piston motion optimization; endoreversible thermodynamics; vuilleumier refrigerator; cooling power optimization; heat-only operation

\section{Introduction}

In Vuilleumier machines [1,2] a working gas is cyclically transferred back and forth between three working spaces, each of which is thermally coupled to a different external heat bath and is thus maintained at a different temperature level. This is schematically shown in Figure 1. The gas transfers are induced by two independently movable displacer pistons. In order to reduce thermal mixing - and the resulting entropy production-during those gas transfers, two regenerators are employed in the ports between the hot and medium as well as the medium and cold working spaces.

In fact, Vuilleumier refrigerators come in different technical configurations. The two regenerators mentioned above often consist of an open-pored metal matrix that is fixed at the main structure of the machine. Here however, we consider a different configuration where they are integrated in the displacer pistons, as depicted in Figure 1. Apart from the regenerator configuration, different types of piston drives are possible. The displacer pistons can, for example, run in different cylinders where they are driven by a mechanical linkage. Alternatively, a free-piston configuration can be used, where the displacers are oscillators constituting a pressure-driven, damped mass-spring system.

In any case, the control of the two displacer pistons is performed in such a way that it leads to the thermal compression and expansion of the working gas, which in turn induces heat fluxes between the three external heat baths and the working spaces. Thus, heat coming from the hot bath can be utilized to extract heat from the cold bath and reject both heats to the medium bath. Correspondingly, the Vuilleumier machine can act as a heat-driven heat pump such as in residential heating [3-5], or it can act as a heat-driven refrigerator for low-temperature cooling [6-10], air conditioning [11], or waste heat-driven cargo cooling such as on fishing vessels [3]. 


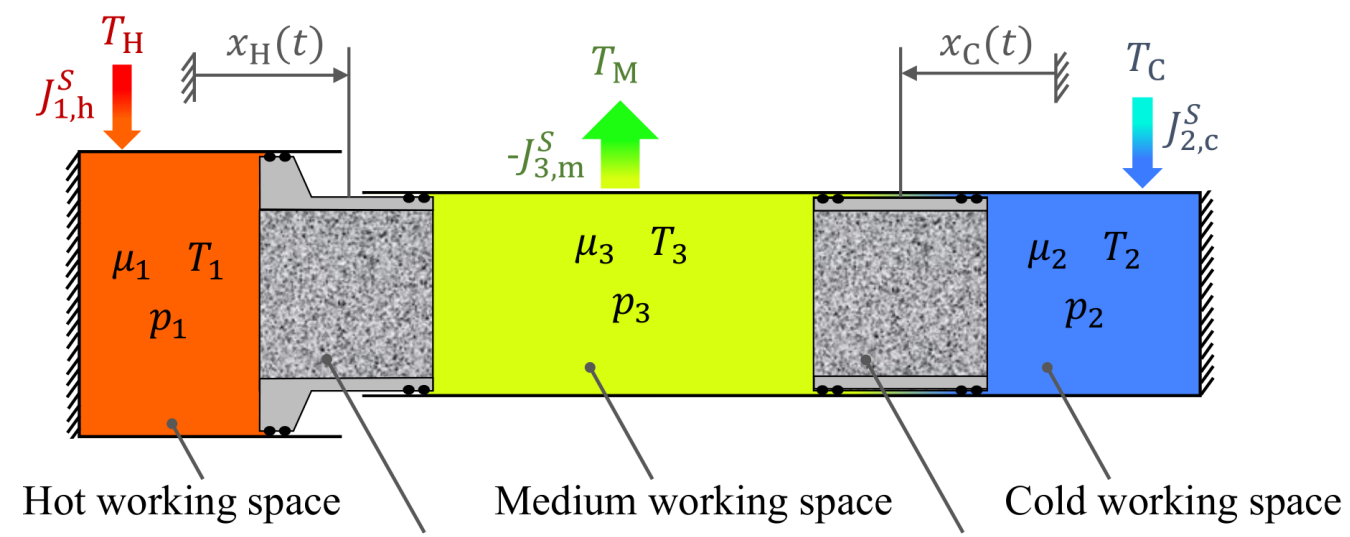

Displacer incl. hot regenerator

Displacer incl. cold regenerator

Figure 1. Schematic representation of a Vuilleumier engine consisting of the three subsystems 1, 2, and 3, which are connected by two displacer pistons with postions $x_{\mathrm{H}}(t)$ and $x_{\mathrm{C}}(t)$. The subsystems represent the working spaces containing the working fluid characterized by its temperature $T_{i}$, its pressure $p_{i}$, and its chemical potential $\mu_{i}$. The working spaces exchange entropy fluxes $J_{1, \mathrm{~h}}^{S} J_{3, \mathrm{~m}}^{S}$, and $J_{2, \mathrm{c}}^{S}$ with external heat baths with temperatures $T_{\mathrm{H}}>T_{\mathrm{M}}>T_{\mathrm{C}}$, respectively. The pistons control the volume of the adjacent subsystems and additionally act as regenerators. Note the surface differences between both ends of the hot-side piston, which is required in order to gain the power to operate the piston drive.

The thermodynamic operation of a Vuilleumier refrigerator can be understood as a combination of a Stirling engine and a Stirling refrigerator in the sense that boththe Vuilleumier refrigerator and the combination-lead to the same overall changes in the environment:

In the combination, the clockwise-run Stirling cycle in the Stirling engine takes heat from the hot bath, disposes it to the medium bath, and produces work. This work is transferred to the counterclockwise-run Stirling cycle in the Stirling refrigerator and is employed there to pump heat from the cold bath to the medium bath. Additionally, part of the work gained in the clockwise-run Stirling cycle may be used to compensate for frictional losses of the displacer pistons and the pressure drop across the regenerators. In the Vuilleumier refrigerator, the described work transfer occurs internally, and the cold side of the driving Stirling engine and the hot side of the refrigerating Stirling engine share the same working space (the medium working space in Figure 1), from which heat is disposed into the environment.

The control (or motion) of the displacer pistons strongly influences these processes. In real Vuilleumier machines the displacer motion is typically close to the harmonic functions of time. This is, however, not necessarily optimal regarding the cooling power or coefficient of performance. In fact, Chen et al. [12,13] showed that significant performance improvements are possible by adapting the displacer piston motion of a Vuilleumier machine. In this particular case, a dwell-based piston motion [14] was used, where the displacers were held in their extreme positions for defined shares of the cycle time. Interestingly, for different Stirling engines, which are thermodynamically closely related to Vuilleumier machines, it was found that power-optimal piston motions tend to involve such piston dwells [15-18].

The aim of this investigation is to determine the possible gain in cooling power by optimizing the displacer piston motion. In particular, we are interested in the dependence of this potential gain on the regeneration extent of the regenerators used as well as on the engine speed.

In order to optimize the piston motion, several possibilities exist. One is to use optimal control theory, which has already been applied successfully to a variety of thermal devices ranging from nano devices [19] to, for instance, diesel [20-22] and light-driven 
engines [23-25]. However, with the increasing complexity of thermal devices, the numerical effort of using control theory is also growing rapidly. In particular, the use of cyclic optimal control theory requires additional considerations with added numerical demands due to the settling times necessary to reach cyclic operation. Thus, in this study, we opt for a novel approach by using, on the one hand, an endoreversible model of limited complexity that captures the main loss mechanisms in a Vuilleumier refrigerator, and on the other hand, the recently introduced AS ("adjustable sinusoidal") motion class for cyclic dynamics [17], which provides a parameterized set of functions for the piston movement. This approach lead to a much lower numerical demand from the optimization procedure already present in our work on the optimized piston motion for a Stirling engine [18]. We note however, that solutions based on the AS motion class give only lower bounds on the performance gains, which might later be determined by control theory for this given engine model. In that sense, our work can provide directions for more elaborate studies in the future.

Our presentation is organized as follows. We start with an introduction to the AS motion class, which will be used for the piston motion. Then, we develop the thermodynamic model of the Vuilleumier refrigerator based on Endoreversible Thermodynamics. In particular, the important loss mechanisms in heat and gas transport as well as frictional losses are considered. We also describe the regeneration process in detail using the $r$-regenerator model developed in [18]. Subsequently, the optimal piston motion is determined and the cooling power gains are studied in relation to the engine speed. Moreover, the changes in the piston motion compared to the standard case are presented. Finally, the design requirements to guarantee a heat-only driven operation of the Vuilleumier refrigerator without auxiliary power are discussed.

\section{The AS Motion Class}

The AS ("adjustable sinusoidal") motion class for cyclic dynamics [17] describes a set of functions, which are parameterized by the two parameters $\sigma$ and $\delta$. All members $f_{\mathrm{AS}}(x ; \sigma, \delta)$ of the class are periodic with period $1: f_{\mathrm{AS}}(x ; \sigma, \delta)=f_{\mathrm{AS}}(x+1 ; \sigma, \delta)$. The motion class is based on the two defining functions

$$
f_{1}(x ; \sigma)=(\sin (2 \pi x+\sigma \sin (4 \pi x))+1) / 2
$$

and

$$
f_{2}(x ; \delta)=x+\delta(1-\cos (2 \pi x))
$$

which are combined to give the AS motion function

$$
f_{\mathrm{AS}}(x ; \sigma, \delta)=f_{1}\left(f_{2}(x ; \delta) ; \sigma\right) .
$$

For our application it is helpful that the AS motion class allows for the recovery of the standard $\sin$ function scaled to values between 0 and 1 by setting $\sigma=\delta=0$. Moreover, to ensure the desired shape and features of $f_{\mathrm{AS}}$, the parameters $\sigma$ and $\delta$ are constrained to the intervals $-0.13<\sigma<0.6$ and $-0.08<\delta<0.08$. The bounds on sigma and delta are chosen such that $f_{\text {AS }}$ features only one interval with values close to its maximum value and one close to its minimum value. In addition, for vanishing delta and minimal sigma the resulting piston motion nearly has constant speed during extended periods of the cycle.

The influence of the two parameters, $\sigma$ and $\delta$, is shown in Figures 2 and 3, respectively. Positive $\sigma$ leads to a prolonged residence time close to the extreme values 0 and 1 , with a corresponding faster movement between these phases. Negative values shorten the periods close to the extreme values. The parameter $\delta$ allows to shift that time, when the start/end value of 0.5 is crossed during the cycle, to shorter or longer times, as can be seen in Figure 3. 
For the configuration of the Vuilleumier refrigerator with its two pistons containing the regenerators chosen here, we set the position of the two pistons $x_{\mathrm{H}}(t)$ and $x_{\mathrm{C}}(t)$ as follows:

$$
\begin{aligned}
& x_{\mathrm{H}}(t)=s f_{\mathrm{AS}}\left(t / t_{0} ; \sigma_{1}, \delta_{1}\right), \\
& x_{\mathrm{C}}(t)=s f_{\mathrm{AS}}\left(t / t_{0}+\Delta ; \sigma_{2}, \delta_{2}\right),
\end{aligned}
$$

where $t_{0}$ is the cycle time, and $s$ is the stroke length of the pistons.

Note the parameter $\Delta$, which facilitates a phase shift between the two piston motions. The standard harmonic dynamics of the Vuilleumier refrigerator has a phase shift of $\pi / 2$ leading to $\Delta=0.25$ and is defined by $\sigma_{1}=\delta_{1}=\sigma_{2}=\delta_{2}=0$.

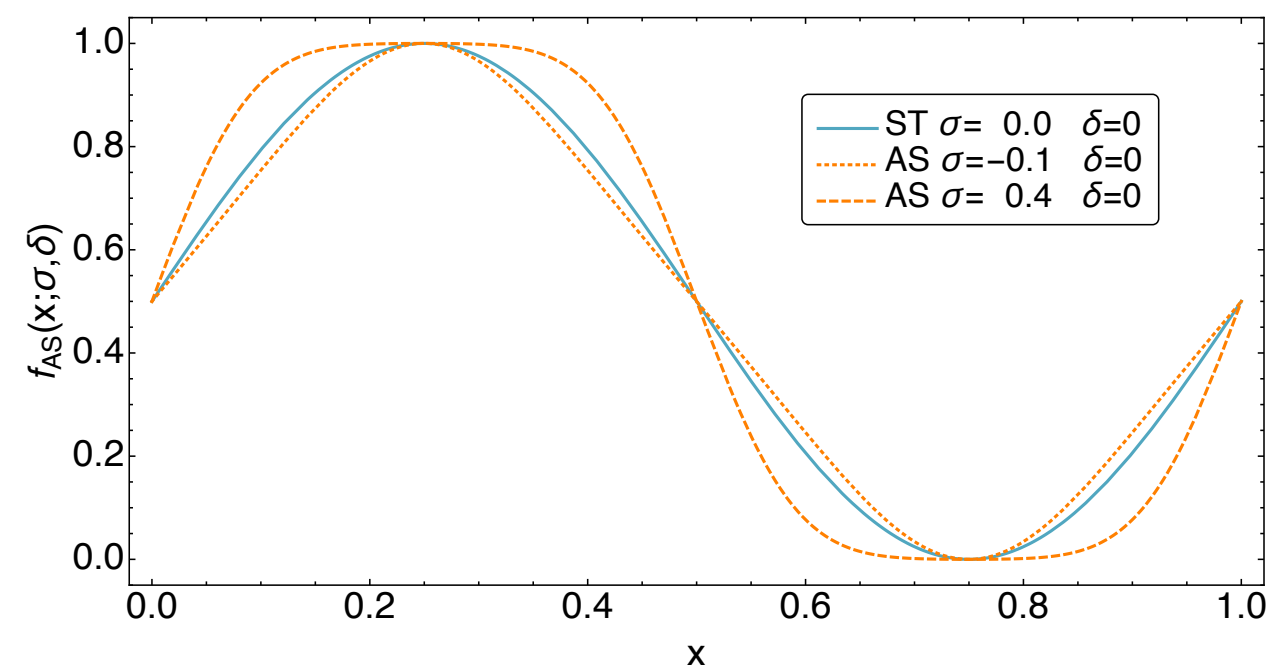

Figure 2. The defining function $f_{\mathrm{AS}}(x ; \sigma, \delta)$ of the AS motion class for three values of the shape parameter $\sigma$ compared to standard sinusoidal motion (ST) at $\sigma=0$. Note that $f_{\mathrm{AS}}(x ; \sigma, \delta)$ is periodic with period 1 as a function of its independent variable $x$. For values $\sigma<0$, the shape of the motion is shifted to be more triangular, while for values $\sigma>0$, the shape becomes increasingly square-like.

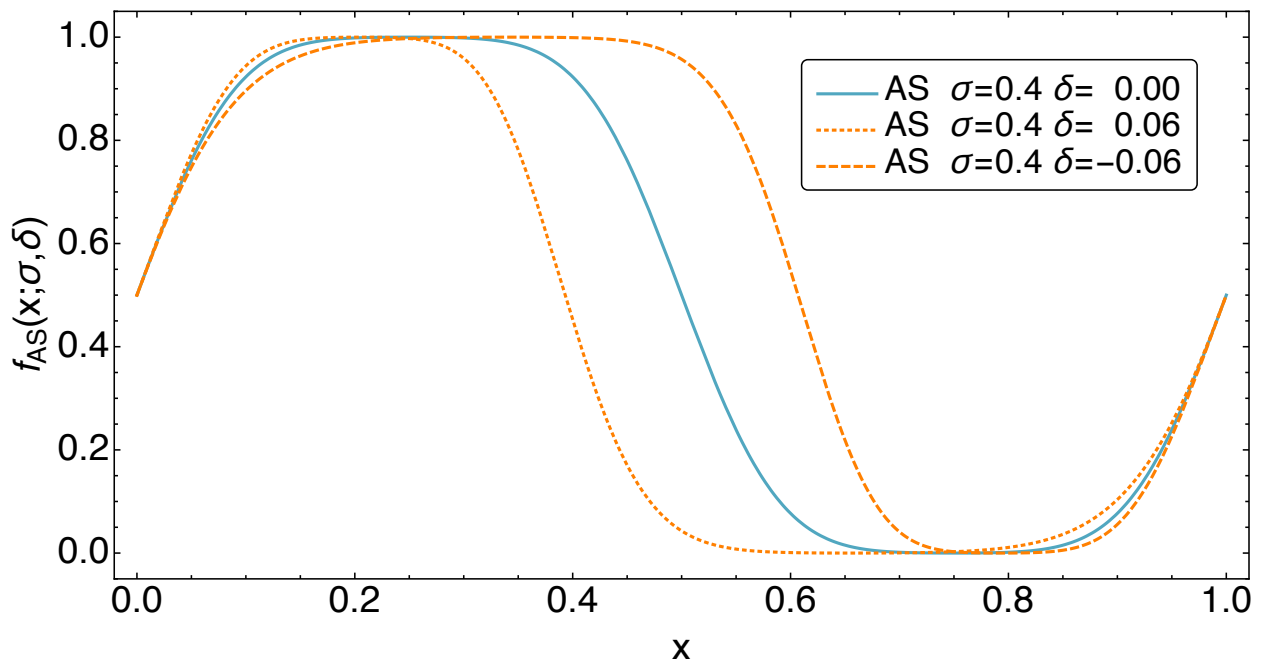

Figure 3. The defining function $f_{\mathrm{AS}}(x ; \sigma, \delta)$ of the AS motion class for three values of the shape parameter $\delta$. This parameter controls the time fraction spent above and below $1 / 2$. Equal times spent within both regions are found at $\delta=0$.

\section{An Endoreversible Model of the Vuilleumier Refrigerator}

Establishing realistic bounds for the performance gains of Vuilleumier refrigerators through an optimized piston motion requires an adequate treatment of the important loss 
terms in a real device. At the same time it is necessary to obtain a numerically efficient model that is suitable for performing optimizations. Therefore, in this study, we use a modeling ansatz for the Vuilleumier refrigerator that is based on Endoreversible Thermodynamics [26-28]. In essence, the idea is that the overall non-equilibrium system can be described as a network of internally reversible ("endoreversible") subsystems that interact in irreversible ways. Thus, the subsystems can be modeled with the tools of equilibrium thermodynamics, while irreversibilities are captured in the transfer laws that constitute the interactions of those subsystems. This network approach leads to a clear model structure and has also been used in other science fields in connection with control problems; for instance, see [29]. The advantages of the endoreversible approach become apparent in different types of thermodynamic systems such as solar-power driven thermal engines [30-33] and chemical devices [34-38]. At the heart of Endoreversibe Thermodynamics are of course heat engines operating at deterministic [39-42] and randomly varying conditions [43-45]. Recent applications of the underlying ideas cover thermoelectric devices [46,47], waste heat recovery [48], and optimization studies [49-52].

The endoreversible approach maps real systems undergoing thermodynamic processes into a network of subsystems, which exchange thermodynamic extensities and the accompanying energy. The extensities of interest here are the entropy $S$, volume $V$, and mol number $n$, but more generally, the extensities (extensive thermodynamic variables) may also include charge, momentum, or angular momentum. If an extensity $\alpha$ is added to a thermodynamic subsystem $i$ by an extensity flux $J_{i, k}^{\alpha}$ through a contact point $(i, k)$, the total content $X_{i}^{\alpha}$ of this extensity in subsystem $i$ can be described by a balance equation:

$$
\dot{X}_{i}^{\alpha}=\sum_{k} J_{i, k}^{\alpha}
$$

where the sum runs over all contact points of extensity $\alpha$ in subsystem $i$. The endoreversibility assumption for a subsystem requires the subsystem to be in thermal equilibrium, which means that its energy can be expressed as a function of its extensive variables [53]:

$$
U_{i}=U_{i}\left(X^{\alpha}\right)
$$

For subsystem $i$, one then knows all intensive thermodynamic variables, referred to here as intensities

$$
Y_{i}^{\alpha}=\frac{\partial U_{i}\left(X_{i}^{\alpha}\right)}{\partial X_{i}^{\alpha}}
$$

through the differentiation of $U$ with respect to the corresponding extensity $X_{i}^{\alpha}$. Subsystems which obey the relations above are referred to as finite reservoirs. As opposed to that, for infinite reservoirs (as for instance an infinite-capacity heat bath), the internal energy is infinite and therefore instead of defining $U_{i}\left(X_{i}^{\alpha}\right)$ the intensity values $Y_{i}^{\alpha}$ are prescribed. In this case, the $Y_{i}^{\alpha}$ are not changed as extensity fluxes enter or exit the infinite reservoir. Finite and infinite reservoirs build a first group of subsystems.

The second major group of subsystems encompasses engines. Endoreversible engines operate reversibly and can redistribute energy "carried" into it by an extensity $X^{\alpha}$ to another extensity. However, contrary to reservoirs, engines have no storage facility for extensities, such that in the model used here the inflows of all extensities have to balance at each moment:

$$
0=\sum_{k} J_{i, k}^{\alpha} \quad \text { for each } \alpha .
$$


The same applies for the accompanying energy fluxes:

$$
0=\sum_{\alpha, k} Y_{i, k}^{\alpha} J_{i, k}^{\alpha}=\sum_{\alpha, k} I_{i, k}^{\alpha}
$$

where we made use of the relation $I_{i, k}^{\alpha}=Y_{i, k}^{\alpha} J_{i, k}^{\alpha}$, which couples an energy flux to its "carrier" extensity flux through the corresponding intensity. Note that for an engine $i$, the intensities $Y_{i, k}^{\alpha}$ associated with an extensity $\alpha$ can be different at each of its contact points $(i, k)$. This is not so for reservoirs, which are usually considered to be homogeneous, thus causing each intensity $Y_{i}^{\alpha}$ to have the same value at all contact points of a reservoir.

Reservoirs and engines are the nodes of the endoreversible network and will be labeled by a sequence of upper case letters or numbers in the endoreversible model developed below. The connections between the nodes are built up by interactions, which describe the transport of extensities and the accompanying energies between the nodes. Interactions will be labeled by sequences $k$ of lower case letters, and we will use those to label the contact points at subsystem $i$ of that interaction by the tuple $(i, k)$.

Typically, an interaction is defined by transport equations such as the Fourier law for heat transport or the diffusion equation for particle transport. Technically, an interaction is defined by specifying its set of contact points and the extensity fluxes at each contact point. Alternatively, as the extensity flux and the corresponding energy flux are coupled by the contact point intensity in $I_{i, k}^{\alpha}=Y_{i, k}^{\alpha} J_{i, k}^{\alpha}$, one can specify the energy flux of the extensity $\alpha$.

Such interactions can be reversible or irreversible, and below we will encounter both cases. In the interactions considered here, all extensities apart from entropy are conserved:

$$
0=\sum_{i} J_{i, k}^{\alpha} \quad \text { for each } k \text { and each } \alpha \text { other than } S .
$$

In a reversible interaction the entropy is also conserved, while in an irreversible interaction entropy will be produced and-as there is no storage for entropy-has to leave the interaction through an entropy contact point. Thus, an entropy contact point must be part of the contact point set of an irreversible interaction. Sometimes, if one is only interested in the energetic features of certain interactions, one does not specify their carrier extensities and thus considers only their energy fluxes, which are then labeled by $P_{i, k}$.

\subsection{The Endoreversible Vuilleumier Refrigerator Model}

In Figure 4 the endoreversible Vuilleumier refrigerator model is shown. It features nine subsystems and six bookkeeping reservoirs. The latter are present to facilitate the bookkeeping of extensities. There are in particular three external heat baths, $\mathrm{H}, \mathrm{M}$, and C, which are infinite capacity reservoirs with constant temperatures $T_{H}, T_{M}$, and $T_{C}$, respectively. "External heat bath" here does not refer to the original source or sink of heat; it instead refers to the input side of the heat exchangers of the Vuilleumier refrigerator. Each of the heat baths is connected by heat conduction to a working space with working gas, which is at a temperature "close" to its corresponding heat bath temperature. We will refer to these finite capacity reservoirs as working spaces 1, 2, and 3, respectively. The two engines TH and TC represent the piston mechanisms, which transform the $p V$-work of the working spaces into work; this is then split into a fraction that covers frictional losses transported to the bookkeeping reservoir WF and a fraction of usable work entering the bookkeeping reservoir WT. In order to balance the volume fluxes in TH and TC, we have an infinite volume reservoir $\mathrm{E}$ with a fixed pressure $p_{\mathrm{E}}$. Finally, there are bookkeeping reservoirs SRH and WRH for the hot regenerator, and SRC and WRC for the cold regenerator. The operation of the regenerators are mapped into two complex interactions rh and rc. For further nomenclature see Figure 4.

In the following detailed description of the model, we will first discuss the interactions; we start with the volume interactions connected to the two engines TH and TC. 


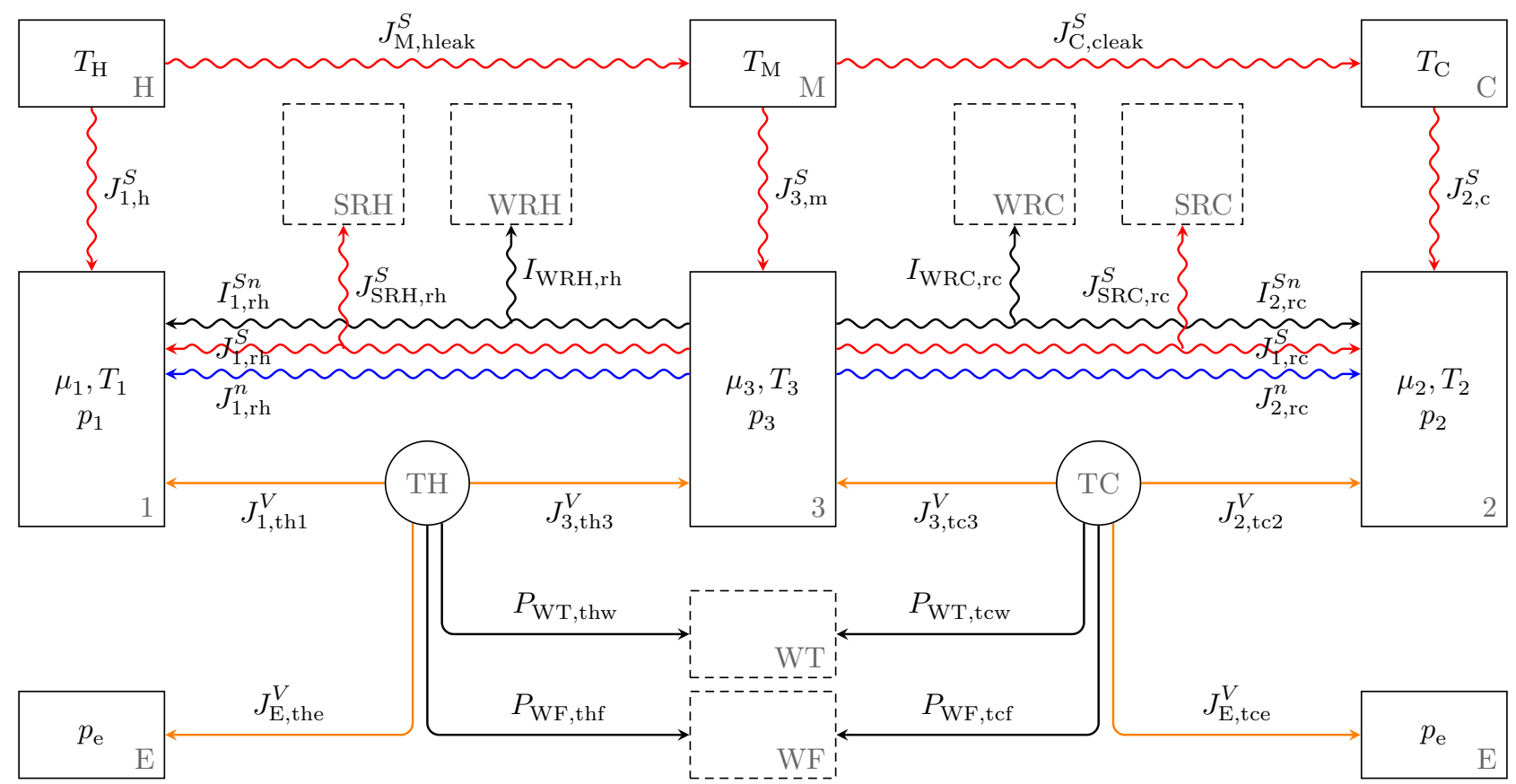

Figure 4. Endoreversible model of the Vuilleumier refrigerator. Note the three external reservoirs $\left(T_{\mathrm{H}}, T_{\mathrm{M}}, T_{\mathrm{C}}\right)$ and the corresponding internal reservoirs $\left(T_{1}, T_{2}, T_{3}\right)$. The two engines $\mathrm{TH}$ and $\mathrm{TC}$ transform the volume work fluxes into power streams entering the bookkeeping reservoirs WT and WF.

\subsubsection{Volume Interactions}

First, we introduce the volume dynamics in the three working spaces based on the AS motion class introduced above. Depending on the cross sectional area of the pistons on their two sides, which can differ from one another as schematized in Figure 1, the position dynamics of the pistons from Equation (4) leads to the following volume dynamics in working spaces 1, 2, and 3:

$$
\begin{aligned}
& V_{1}(t)=V_{0}+D_{1} f_{\mathrm{AS}}\left(t / t_{0} ; \sigma_{1}, \delta_{1}\right), \\
& V_{2}(t)=V_{0}+D_{2} f_{\mathrm{AS}}\left(t / t_{0}+\Delta ; \sigma_{2}, \delta_{2}\right),
\end{aligned}
$$

where $V_{0}$ is the dead volume, and the $D_{1}$ and $D_{2}$ are the respective displacements. Working space 3 is virtually split into two parts facing working spaces 1 and 2:

$$
\begin{aligned}
V_{31}(t) & =V_{0} / 2+D_{31}\left(1-f_{\mathrm{AS}}\left(t / t_{0} ; \sigma_{1}, \delta_{1}\right)\right), \\
V_{32}(t) & =V_{0} / 2+D_{32}\left(1-f_{\mathrm{AS}}\left(t / t_{0}+\Delta ; \sigma_{2}, \delta_{2}\right),\right. \\
V_{3}(t) & =V_{31}(t)+V_{32}(t) .
\end{aligned}
$$

Based on the volume dynamics, we can now specify the volume interactions. On the hot side of the Vuilleumier refrigerator, these are the volume exchanges between working spaces 1 and 3, the environment E, and engine TH. These interactions are reversible; thus, for each interaction the pressure at its contact point of the engine is the same as at the contact points of the other connected subsystem. Their volume fluxes are

$$
J_{1, \text { th1 }}^{V}=\dot{V}_{1}(t)=-J_{\mathrm{TH}, \mathrm{th} 1}^{V} \quad J_{3, \text { th } 3}^{V}=\dot{V}_{31}(t)=-J_{\mathrm{TH}, \text { th } 3}^{V} .
$$


Applying Equation (8) for the engine TH to the extensity "volume", one finds $J_{\mathrm{TH}, \mathrm{th} 1}^{V}+$ $J_{\mathrm{TH}, \mathrm{th} 3}^{V}+J_{\mathrm{TH}, \text { the }}^{V}=0$ and thus

$$
J_{\mathrm{TH}, \text { the }}^{V}=-J_{\mathrm{TH}, \mathrm{th} 1}^{V}-J_{\mathrm{TH}, \mathrm{th} 3}^{V}=-J_{\mathrm{E}, \text { the }}^{V} .
$$

The energy harvested from these volume interactions is split into two "pure" energy fluxes $P_{\mathrm{WF}, \text { thf }}$ and $P_{\mathrm{WT}, \text { thw }}$. The first one captures the frictional losses due to the piston movement and that of connected parts, which are here modeled as follows:

$$
P_{\mathrm{WF}, \mathrm{thf}}=\beta \dot{V}_{31}^{2} \text {. }
$$

These will be dissipated to heat outside of our model. The remaining pure energy flux $P_{\mathrm{WT}, \text { thw }}$ follows from Equation (9):

$$
P_{\mathrm{WT}, \text { thw }}=-P_{\mathrm{WF}, \mathrm{thf}}+I_{\mathrm{TH}, \mathrm{th} 1}^{V}+I_{\mathrm{TH}, \mathrm{th} 3}^{V}+I_{\mathrm{TH}, \text { the }}^{V} .
$$

We finish this section by pointing out that the interactions on the cold side of the Vuilleumier refrigerator are treated in exactly the same way by replacing the temperature label " $\mathrm{H}$ " or " $\mathrm{h}$ " with " $\mathrm{C}$ " or " $\mathrm{c}$ ", and the working space label " 1 " with " 2 ". This also applies to the following discussion of the regenerator interactions.

\subsubsection{The Regenerators}

Regenerators are devices which cyclically take up heat from hot streams of working gas and provide it to cold streams of working gas. Thus, they improve the efficiency of heat engines or refrigerators by avoiding the discharge of temporarily unused energy. As usual, the regeneration processes require finite energy fluxes; thus, dissipation is practically inevitable. In fact, the regenerator's performance depends on a variety of features and is degraded by several loss mechanisms. For example, such losses have been designated as reheat loss, temperature swing loss, thermal conduction loss, internal temperature swing loss, dispersion loss, and bypass loss [54].

For the energetic balance of the regeneration process the important question is to what extent the withdrawal of energy from and its subsequent addition to the gas flow succeeds. Based on the assumption that the gas content inside the regenerator is of minor importance in the description and can thus be neglected, the $r$-regenerator model was developed in [18]. We will use this model here as well. It captures the effects of regeneration in the form of an irreversible interaction. For more elaborate models see $[55,56]$.

We start with the observation that gas leaving the hot working space and entering the regenerator has the properties of the gas in the source working space, while the gas leaving the regenerator and entering the sink working space will typically have properties in between those of the hot and the cold working space.

Let us consider the hot regenerator operating between working spaces 1 and 3 . This interaction is labeled rh and describes the gas flow as a multi-extensity flux [37]. The two extensities in this flux are particles (mols) of working fluid and entropy. The particle fluxes are assumed to be proportional to the pressure difference:

$$
J_{1, \mathrm{rh}}^{n}=\alpha\left(p_{3}-p_{1}\right)=-J_{3, \mathrm{rh}}^{n},
$$

where $\alpha$ is the mass transfer coefficient, which shall have the same value in the cold regenerator.

As already pointed out above, the flow direction of the gas in the regenerator is important. We will thus write fluxes as the sum of two parts, one which corresponds to a positive mol number flux, and one which which corresponds to a negative mol number flux:

$$
\begin{aligned}
J_{i, \mathrm{r}}^{\alpha} & =J_{\rightarrow i, \mathrm{r}}^{\alpha}+J_{\leftarrow i, \mathrm{r}}^{\alpha}, \\
I_{i, \mathrm{r}}^{\alpha} & =I_{\rightarrow i, \mathrm{r}}^{\alpha}+I_{\leftarrow i, \mathrm{r}}^{\alpha},
\end{aligned}
$$


where $J_{\rightarrow i, \mathrm{r}}^{\alpha}$ and $I_{\rightarrow i, \mathrm{r}}^{\alpha}$ apply when $J_{i, \mathrm{r}}^{n}$ is positive and gas flows into reservoir $i$, while $J_{\leftarrow i, \mathrm{r}}^{\alpha}$ and $I_{\leftarrow i, \mathrm{r}}^{\alpha}$ apply when $J_{i, \mathrm{r}}^{n}$ is negative and gas flows out of reservoir $i$.

For instance, for the energy flux carried by the multi-extensity gas flow this leads to

$$
I_{\leftarrow 1, \mathrm{rh}}^{S n}= \begin{cases}J_{1, \mathrm{rh}}^{n} h_{1} & \text { if } \quad J_{1, \mathrm{rh}}^{n}<0, \\ 0 & \text { otherwise, }\end{cases}
$$

where $h_{i}=H_{i} / n_{i}$ is the molar enthalpy of subsystem $i$. For the other flow direction, this leads to

$$
I_{\rightarrow 1, \mathrm{rh}}^{S n}\left(r_{\mathrm{h} 1}\right)= \begin{cases}J_{1, \mathrm{rh}}^{n}\left(r_{\mathrm{h} 1} h_{1}+\left(1-r_{\mathrm{h} 1}\right) h_{3}\right) & \text { if } J_{1, \mathrm{rh}}^{n}>0 \\ 0 & \text { otherwise }\end{cases}
$$

where $r_{\mathrm{h} 1}$ is the hot regeneration coefficient for working space 1 characterizing the regeneration extent. It determines the properties of the gas entering the sink working space.

For the fluxes at working space 3 , one sets the following:

$$
I_{\leftarrow 3, \mathrm{rh}}^{S n}= \begin{cases}J_{3, \mathrm{rh}}^{n} h_{3} & \text { if } J_{3, \mathrm{rh}}^{n}<0, \\ 0 & \text { otherwise, }\end{cases}
$$

and

$$
I_{\rightarrow 3, \mathrm{rh}}^{S n}\left(r_{\mathrm{h} 3}\right)= \begin{cases}J_{3, \mathrm{rh}}^{n}\left(r_{\mathrm{h} 3} h_{3}+\left(1-r_{\mathrm{h} 3}\right) h_{1}\right) & \text { if } J_{3, \mathrm{rh}}^{n}>0, \\ 0 & \text { otherwise }\end{cases}
$$

where $r_{\mathrm{h} 3}$ is the hot regeneration coefficient for working space 3 .

As in real operation, the gas flow leaving a working space thus has the properties of the compartment it comes from, while the properties of a flow entering a working space come closer to those of the receiving compartment with better regeneration. For a perfect regeneration $\left(r_{\mathrm{h} 1}=r_{\mathrm{h} 3}=1\right)$, the flow would have exactly those of the receiving one. For $r_{\mathrm{h} 1}=r_{\mathrm{h} 3}=0$, the gas would enter the receiving compartment with the unchanged properties of the origin compartment.

The total energy flux of this multi-extensity interaction at a contact point is the sum of the two energy fluxes carried by the particle flux and the entropy flux:

$$
I_{i, \mathrm{rh}}^{S n}=I_{i, \mathrm{rh}}^{n}+I_{i, \mathrm{rh}}^{S}
$$

which allows for the identification of the entropy flux. Using $I_{i, k}^{\alpha}=Y_{i, k}^{\alpha} J_{i, k}^{\alpha}$, one finds for incoming fluxes:

$$
J_{\rightarrow i, \text { rh }}^{S}=\left(I_{\rightarrow i, \text { rh }}^{S n}-\mu_{i} J_{\rightarrow i, \text { rh }}^{n}\right) / T_{i},
$$

where $\mu_{i}=Y_{i}^{n}$ is the chemical potential in subsystem $i$, and for outgoing fluxes:

$$
J_{\leftarrow i, \mathrm{rh}}^{S}=s_{i} J_{\leftarrow i, \mathrm{rh}}^{n},
$$

where $s_{i}=S_{i} / n_{i}$ is the molar entropy of subsystem $i$.

Finally, the fluxes into the bookkeeping reservoirs WRH and SRH are defined as

$$
\begin{aligned}
I_{\mathrm{WRH}, \mathrm{rh}} & =-I_{1, \mathrm{rh}}^{S n}-I_{3, \mathrm{rh}}^{S n} \quad \text { and } \\
J_{\mathrm{SRH}, \mathrm{rh}}^{S} & =-J_{1, \mathrm{rh}}^{S}-J_{3, \mathrm{rh}}^{S} .
\end{aligned}
$$

For cyclic operation with cycle time $t_{0}$, these allow for the definition of the cycleaveraged energy flux from regenerator RH in the bookkeeping reservoir WRH. By applying the requirement, that in any interaction energy must be conserved, to the energy fluxes of 
the rh-interaction (see Figure 4), we find $0=I_{\mathrm{WRH}, \mathrm{rh}}+I_{1, \mathrm{rh}}^{s n}+I_{3, \mathrm{rh}}^{s n}$. Solving for $I_{\mathrm{WRH}, \mathrm{rh}}$ and splitting $I_{1, \mathrm{rh}}^{s n}$ and $I_{3, \text { rh }}^{s n}$ according to Equation (22), we arrive at the following:

$$
P_{\mathrm{RH}}=\frac{1}{t_{0}} \int_{0}^{t_{0}} I_{\mathrm{WRH}, \mathrm{rh}} \mathrm{d} t=\frac{-1}{t_{0}} \int_{0}^{t_{0}}\left(I_{\leftarrow 1, \mathrm{rh}}^{S n}+I_{\leftarrow 3, \mathrm{rh}}^{S n}+I_{\rightarrow 1, \mathrm{rh}}^{S n}\left(r_{\mathrm{h} 1}\right)+I_{\rightarrow 3, \mathrm{rh}}^{S n}\left(r_{\mathrm{h} 3}\right)\right) \mathrm{d} t .
$$

The cycle-averaged energy flux $P_{\mathrm{RH}}$ depends on the regeneration coefficients $r_{\mathrm{h} 1}$ and $r_{\mathrm{h} 3}$ for the hot $r$-regenerator [18] and must obey $P_{\mathrm{RH}}=0$ in order to insure the cyclicity of the regenerator. Based on that relation, for a given regeneration coefficient $r_{\mathrm{h}}$ of the hot regenerator, the values of $r_{\mathrm{h} 1}$ and $r_{\mathrm{h} 3}$ are chosen so that the conditions $P_{\mathrm{RH}}=0$ and $r_{\mathrm{h}}=\left(r_{\mathrm{h} 1}+r_{\mathrm{h} 3}\right) / 2$ hold true.

\subsection{Heat Transfer and Heat Leaks}

The Vuilleumier refrigerator operates between the three heat baths $\mathrm{H}, \mathrm{M}$, and $\mathrm{C}$, which represent the input side of the refrigerator's heat exchanges. The heat transport from there into and out of the refrigerator working spaces is modeled to be Newtonian. These heat fluxes are described by the interactions $h, m$, and $c$, for which the energy fluxes at the contact points are

$$
\begin{gathered}
I_{1, \mathrm{~h}}^{S}=\kappa\left(T_{\mathrm{H}}-T_{1}\right)=-I_{\mathrm{H}, \mathrm{h}}^{S}, \\
I_{3, \mathrm{~m}}^{S}=\kappa\left(T_{\mathrm{M}}-T_{3}\right)=-I_{\mathrm{M}, \mathrm{m}}^{S}, \\
I_{2, \mathrm{c}}^{S}=\kappa\left(T_{\mathrm{C}}-T_{2}\right)=-I_{\mathrm{C}, \mathrm{c}}^{S} .
\end{gathered}
$$

Here, $\kappa$ is the heat conductance, which is assumed to be the same for all three interactions. The ensuing entropy fluxes are

$$
\begin{array}{rlrl}
J_{1, \mathrm{~h}}^{S} & =I_{1, \mathrm{~h}}^{S} / T_{1}, & J_{\mathrm{H}, \mathrm{h}}^{S}=I_{\mathrm{H}, \mathrm{h}}^{S} / T_{\mathrm{H}}, \\
J_{3, \mathrm{~m}}^{S}=I_{3, \mathrm{~m}}^{S} / T_{3}, & J_{\mathrm{M}, \mathrm{m}}^{S}=I_{3, \mathrm{~m}}^{S} / T_{\mathrm{M}}, \\
J_{2, \mathrm{c}}^{S}=I_{2, \mathrm{c}}^{S} / T_{2}, & J_{\mathrm{C}, \mathrm{c}}^{S}=I_{\mathrm{C}, \mathrm{c}}^{S} / T_{\mathrm{C}} .
\end{array}
$$

While the above heat fluxes are intentional, there are are further heat fluxes which are not. In any real refrigerator, heat leaks are present where energy flows from hotter parts of the device towards colder parts. Such heat leaks are unavoidable even though they can be somewhat diminished by appropriate insulation. However, that is not always possible; the cylinder tubes of the Vuilleumier refrigerator need to sustain pressures on the $100 \mathrm{bar}$ scale and are thus built with metal. In general, heat leaks may be due to heat conduction in the cylinder walls, the regenerators, or other structural elements connecting the hot and cold parts of the refrigerator and can have a decisive influence on the performance of thermodynamic devices $[39,41,57,58]$. Such losses are independent of the engine speed and will be modeled here by the two heat fluxes

$$
\begin{gathered}
I_{\mathrm{M} \text {,hleak }}^{S}=\kappa_{\text {Leak }}\left(T_{\mathrm{H}}-T_{\mathrm{M}}\right)=q_{\text {hLeak }}, \\
I_{\mathrm{C} \text {, cleak }}^{S}=\kappa_{\text {Leak }}\left(T_{\mathrm{M}}-T_{\mathrm{C}}\right)=q_{\text {cLeak }}
\end{gathered}
$$

which capture the heat leak losses due to heat transport inside the refrigerator in an acrossthe-board fashion. We note that these losses are independent of the engine operation and thus coincide with their cycle-averaged values $q_{\text {hLeak }}$ and $q_{\text {cLeak }}$.

Based on all of the introduced extensity fluxes above, we can now determine the thermal dynamics of variables describing the working fluid in the working spaces.

\subsection{Thermal Dynamics of Working Spaces 1, 2, and 3}

In the three reservoirs representing the three working spaces, the thermodynamics is determined by the physical properties of the gas used as working fluid. Here, we 
have chosen to use an ideal gas with a given molar heat capacity $\hat{c}_{V} R$, where $\hat{c}_{V}$ is the dimensionless specific heat capacity at constant volume and $R$ is the gas constant. For the ideal gas, the thermal equation of state is

$$
p V=n R T,
$$

where $p, V, n$, and $T$ are the pressure, volume, mole number, and temperature, respectively. The caloric equation of state is

$$
U=\hat{c}_{V} n R T,
$$

with $U$ being the internal energy.

For the working fluid considered here, the internal energy can be expressed in terms of its natural extensities $S, V$, and $n$. However, any other combination of three variables containing at least one extensity out of the intensities $T, p, \mu$-with $\mu$ being the chemical potential-and the extensities $S, V, n$ and energy $U$ suffice to describe the state of the working gas. Here, we will use $U, V$, and $n$ as independent variables. Then, the other variables can be expressed in those terms. The entropy reads as follows:

$$
S(U, V, n)=n R\left(\hat{c}_{V} \ln \frac{U}{U_{0}}+\ln \frac{V}{V_{0}}-\left(1+\hat{c}_{V}\right) \ln \frac{n}{n_{0}}\right)+n \frac{S_{0}}{n_{0}}
$$

with the reference entropy $S_{0}\left(U_{0}, V_{0}, n_{0}\right)$ and $U_{0}, V_{0}$, and $n_{0}$ denoting the reference internal energy, volume, and mole number, respectively. The intensities have the following form:

$$
\begin{aligned}
T(U, V, n) & =\frac{U}{\hat{c}_{V} n R}, \\
p(U, V, n) & =\frac{U}{\hat{c}_{V} V}, \\
\mu(U, V, n) & =\frac{U}{\hat{c}_{V} n}\left(1+\hat{c}_{V}+\left(1+\hat{c}_{V}\right) \ln \frac{n}{n_{0}}-\hat{c}_{V} \ln \frac{U}{U_{0}}-\ln \frac{V}{V_{0}}-\frac{S_{0}}{n_{0} R}\right) .
\end{aligned}
$$

For given volume fluxes $J_{1, \text { th } 1,}^{V} J_{2, \mathrm{tc} 2}^{V}, J_{3, \text { th } 3}^{V}$, and $J_{3, \mathrm{tc} 3}^{V}$, we can now-based on the balance Equations (8) and (9) applied to working spaces 1, 2, and 3-determine the evolution equations for the respective extensity contents of working spaces 1 and 2 :

$$
\begin{array}{ll}
\dot{U}_{1}=I_{1, \mathrm{~h}}^{S}+I_{1, \mathrm{rh}}^{S}+I_{1, \mathrm{rh}}^{n}+I_{1, \mathrm{th} 1,}^{V} & \dot{U}_{2}=I_{2, \mathrm{c}}^{S}+I_{2, \mathrm{rc}}^{S}+I_{2, \mathrm{rc}}^{n}+I_{2, \mathrm{tc} 2}^{V}, \\
\dot{V}_{1}=J_{1, \mathrm{th} 1,}^{V} & \dot{V}_{2}=J_{2, \mathrm{tc} 2}^{V} \\
\dot{n}_{1}=J_{1, \mathrm{rh},}^{n} & \dot{n}_{2}=J_{2, \mathrm{rc} \prime}^{n}
\end{array}
$$

as well as for working space 3:

$$
\begin{aligned}
& \dot{U}_{3}=I_{3, \mathrm{~m}}^{S}+I_{3, \mathrm{rh}}^{S}+I_{3, \mathrm{rh}}^{n}+I_{3, \mathrm{th} 3}^{V}+I_{3, \mathrm{rc}}^{S}+I_{3, \mathrm{rc}}^{n}+I_{3, \mathrm{tc} 3}^{V} \\
& \dot{V}_{3}=J_{3, \mathrm{th} 3}^{V}+J_{3, \mathrm{tc} 3}^{V} \\
& \dot{n}_{3}=J_{3, \mathrm{rh}}^{n}+J_{3, \mathrm{rc}}^{n} .
\end{aligned}
$$

\section{Cycle-Averaged Energy Fluxes and COPs}

In our study, we are primarily interested in maximizing the cooling power of the Vuilleumier refrigerator. To be specific, we use the cycle-averaged cooling power

$$
q_{\mathrm{C}}=\frac{1}{t_{0}} \int_{0}^{t_{0}} I_{2, \mathrm{c}}^{S} \mathrm{~d} t
$$


as our performance criterion. We will also need the cycle-averaged mechanical power input

$$
P_{\text {aux }}=-\frac{1}{t_{0}} \int_{0}^{t_{0}} P_{\mathrm{WT}, \text { thw }}+P_{\mathrm{WT}, \mathrm{tcw}} \mathrm{d} t
$$

as well as the cycle-averaged heat flux

$$
q_{\mathrm{H}}=\frac{1}{t_{0}} \int_{0}^{t_{0}} I_{1, \mathrm{~h}}^{S} \mathrm{~d} t
$$

entering the Vuilleumier refrigerator in the discussion below.

These cycle-averaged energy fluxes allow us to define an exergetic COP in which the heat input is scaled with the Carnot efficiency $\eta_{\mathrm{C}}=1-T_{\mathrm{M}} / T_{\mathrm{H}}$, which would apply to a heat engine operating between the hot heat bath $\mathrm{H}$ and the intermediate bath $\mathrm{M}$. The COP puts the cycle-averaged cooling power $q_{\mathrm{C}}$ into relation with the exergy influx from heat bath $\mathrm{H}$ and a potentially present auxiliary power input. For vanishing heat leaks, this leads to the following definition:

$$
\mathrm{COP}=\frac{q_{\mathrm{C}}}{\eta_{\mathrm{C}} q_{\mathrm{H}}+P_{\mathrm{aux}}} .
$$

This choice of an exergetic COP allows for a direct comparison with power-driven refrigerators because the driving power is measured on the basis of its exergy content. In the presence of heat leaks, the cycle-averaged cooling power is diminished by the leak flux $q_{\text {cLeak }}$, as can be seen in Figure 4 . With an unchanged operation, the leak flux $q_{\text {cLeak }}$ becomes part of the cooling load and the corresponding amount the cooling power thus decreases. Furthermore, with an unchanged operation, the heat input needs to be increased by an additional heat input compensating $q_{\mathrm{hLeak}}$, which results in the following definition:

$$
\mathrm{COP}_{\text {Leak }}=\frac{q_{\mathrm{C}}-q_{\text {cLeak }}}{\eta_{\mathrm{C}}\left(q_{\mathrm{H}}+q_{\text {hLeak }}\right)+P_{\text {aux }}} .
$$

\section{Heat-Only Driven Vuilleumier Refrigeration}

Our focus in this investigation is on the performance features of a Vuilleumier refrigerator with limited regeneration in a heat-only driven operation mode. With the configuration shown in Figure 1, such an operation mode is not possible for pistons with the same crosssectional area on all four sides. In order to overcome that limitation, we will adapt the displacement $D_{1}$ of working space 1 (for instance by changing its cross-sectional area) while keeping the displacements for working spaces 2 and 3 fixed such that no auxiliary power influx is needed, i.e., we require:

$$
P_{\text {aux }}=0 .
$$

This requirement is implemented by adjusting $D_{1}$ for a given piston motion until $P_{\text {aux }}=0$ is met.

\section{Results}

In order to determine the possible cooling power gains of Vuilleumier refrigerators by optimizing the piston motion, we determined the optimized piston motion using the AS motion class. The results for the optimized motion will be labeled as "OS" (optimized sinusoidal). We will compare these results with those of the standard motion, which will be labeled as "ST". To be more specific, for each set of motion parameters, regeneration coefficients, and the displacement for working space $1\left\{\sigma_{1}, \delta_{1}, \sigma_{2}, \delta_{2}, \Delta, r_{\mathrm{h} 1}, r_{\mathrm{h} 3}, r_{\mathrm{c} 2}, r_{\mathrm{c} 3}, D_{1}\right\}$, the dynamics given by Equations (47)-(52) is integrated numerically until cyclic behavior has been reached. Then, needed bookkeeping quantities are determined. The optimal piston motion is determined by changing the motion parameters $\sigma_{1}, \delta_{1}, \sigma_{2}, \delta_{2}$, and $\Delta$ until the cooling power increases no further. We stress that for each set of motion parameters, cyclic 
operation needs to be established, including the adjustment of the regeneration coefficients and the displacement $D_{1}$. The procedure is based on the Nelder-Mead algorithm [59] and uses parallel execution with runtimes on the order of an hour for one data point in the OS-motion graphs.

The refrigerator parameters are chosen to reflect results for a few $\mathrm{kW}$ of cooling power. They are: $T_{\mathrm{H}}=300^{\circ} \mathrm{C}, \mathrm{T}_{\mathrm{M}}=25^{\circ} \mathrm{C}, \mathrm{T}_{\mathrm{C}}=0{ }^{\circ} \mathrm{C}, t_{0}=0.1 \mathrm{~s}, n_{0}=5 \mathrm{~mol}, V_{0}=0.1 \mathrm{~L}$, $D_{2}=D_{31}=D_{32}=1 \mathrm{~L}, \hat{c}_{V}=5 / 2$. Moreover, we have three parameters describing the non-equilibrium features of the refrigerator: These are the heat conduction coefficient $\kappa_{0}=20 \mathrm{~kW} / \mathrm{K}$, the mass transport coefficient $\alpha_{0}=300 \mathrm{~mol} /(\mathrm{s} \mathrm{bar})$, and the friction coefficient $\beta_{0}=20 \mathrm{kJs} / \mathrm{m}^{6}$. With this choice, the cooling power does not increase much further for enlarged heat conduction and mass transport. We will refer to this parameter set together with $\kappa_{\text {Leak }}=0 \mathrm{~W} / \mathrm{K}$ as the "base case", and with $\kappa_{\text {Leak }}=5 \mathrm{~W} / \mathrm{K}$ as the "standard case".

\subsection{Optimized Piston Motion: Cooling Power}

Our first analysis is focused on the optimized cooling power. We have analyzed a given Vuilleumier refrigerator, for which we determined the cycle-averaged cooling power $q_{C}$ as a function of the engine speed. Of course, the cooling power will depend on the regeneration ability of the refrigerator. We thus analyzed the cooling power and other quantities for two different regeneration abilities: One for high regeneration with regeneration coefficients $r_{\mathrm{h}}=r_{\mathrm{c}}=0.9$ and one for low regeneration with regeneration coefficients $r_{\mathrm{h}}=r_{\mathrm{c}}=0.8$.

The results for the optimized piston motion OS and the high regeneration case are shown on the left in Figure 5. Starting at an engine speed of $100 \mathrm{rpm}$, the cooling power increases to its maximum at around $450 \mathrm{rpm}$ and falls off with a further increase in speed. Beyond $750 \mathrm{rpm}$, the cooling power becomes negative. The general shape of the cooling power roughly follows a parabola. This also applies to the results of the standard harmonic piston motion. The maximum cooling power is reached at about $500 \mathrm{rpm}$, which is only slightly above the OS maximum. The overall gain of cooling power by optimizing the piston motion is more than $15 \%$ compared to the maximum for the standard motion as well as to the standard motion at the same engine speed.

For the low-regeneration case, the general shape of the cooling power results is similar, as shown on the right in Figure 5. However, the cooling power is reduced to about $70 \%$ of the high-regeneration case for the OS motion and 60\% for the ST case. The OS maximum is achieved at about $350 \mathrm{rpm}$, and the ST maximum at a slightly higher speed. For the lowregeneration case, the relative gain of about $30 \%$ due to the optimized motion is however much larger.

\subsection{Optimized Piston Motion: Efficiency at Maximum Cooling Power}

The efficiencies of the Vuilleumier refrigerator at maximum cooling power is shown for the AS and ST motions for both regeneration cases in Figure 6. The efficiencies are measured by the exergetic COP defined above with $P_{\text {aux }}=0 \mathrm{~kW}$ as we consider heat-only operations. In all cases, the COPs fall off from their maximum at $100 \mathrm{rpm}$ in a roughly linear fashion to values close to zero at $750 \mathrm{rpm}$ (OS) and $500 \mathrm{rpm}$ (ST), respectively. With increasing engine speed, the OS cases fall off faster than the ST cases, and for high regeneration, the OS COP is practically equal to the ST COP. While with high regeneration the COPs are in the range between 0.2 and 1.5, the low regeneration COPs are much smaller, with a range between 0 and 0.7 . This very clearly shows that the regeneration extent plays an important role in the efficient operation of the Vuilleumier refrigerator. 

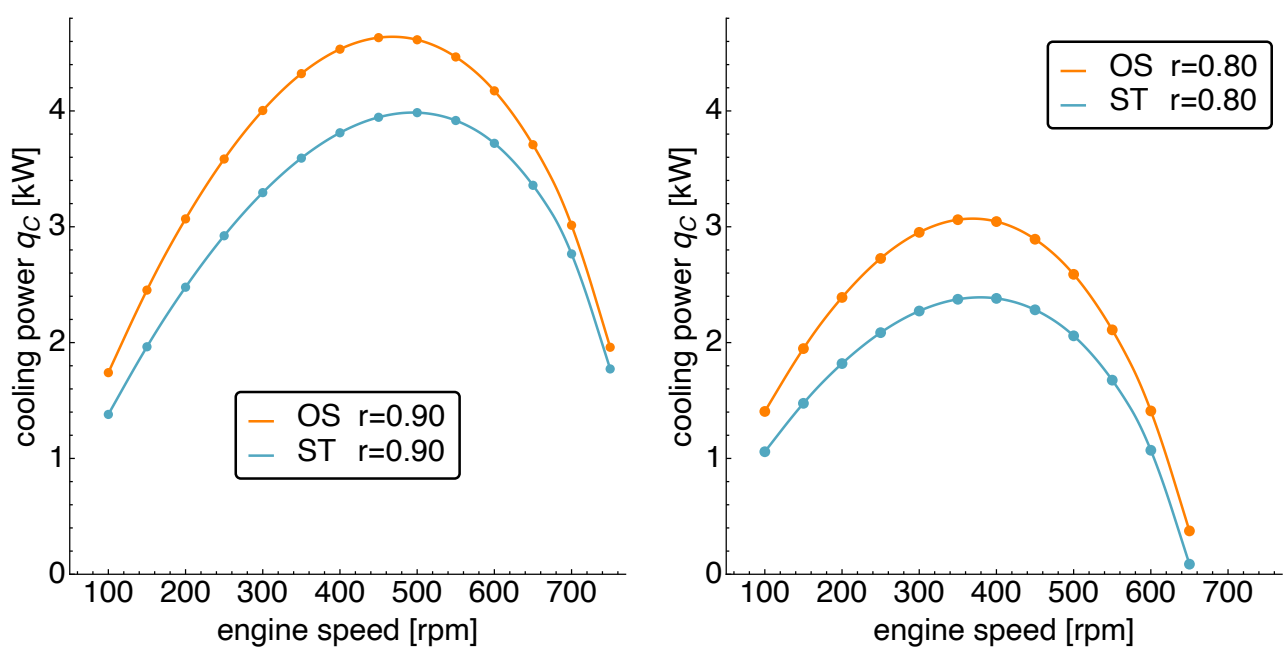

Figure 5. The cooling power $q_{C}$ vs. engine speed for OS and ST motion at different values of the regeneration coefficient (left/right). Comparing the cooling power for $r=0.9$ (left) and $r=0.8$ (right), it is apparent that the larger regeneration coefficient allows for a considerably higher cooling power. We also note that optimizing the piston motion (OS) causes a substantial increase in the cooling power, especially at intermediate speeds for both regeneration cases.
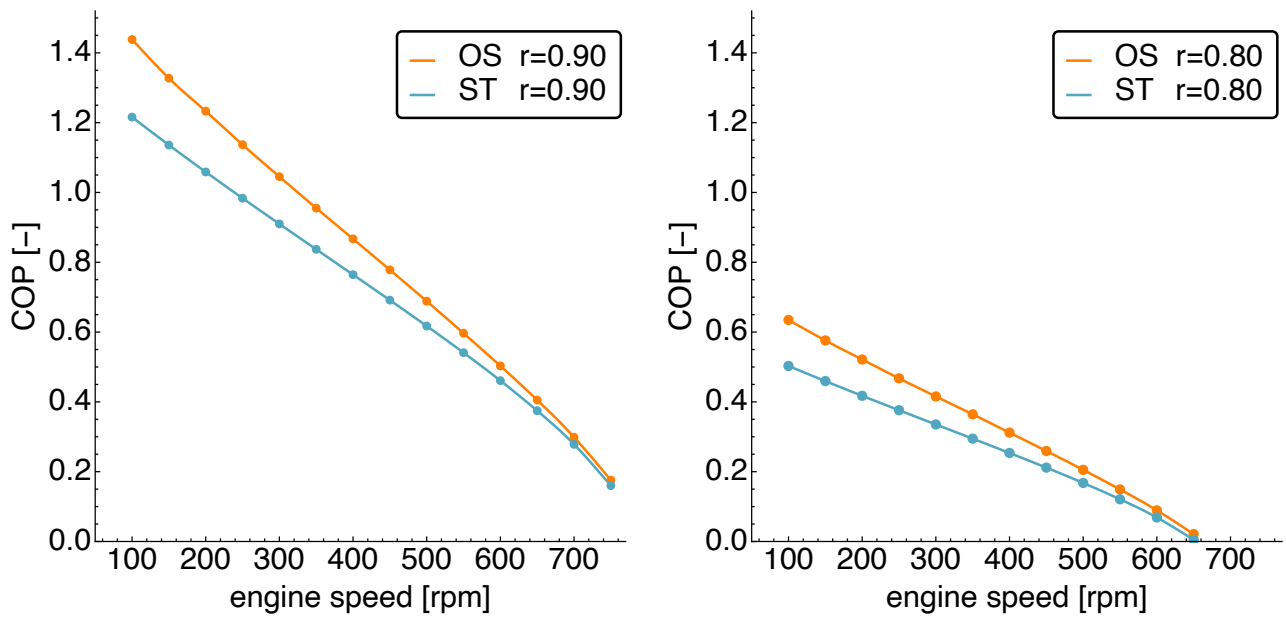

Figure 6. The base case COP vs. engine speed for OS and ST motion at different values of the regeneration coefficient (left/right). The larger regeneration coefficient leads to roughly doubled $\mathrm{COP}$ values as compared to the lower regeneration case. We note that the slower the engine operates, the larger the COP gain by optimized piston motion (OS) is.

\subsection{Heat Leak: Efficiency at Maximum Cooling Power}

In oder to show the influence of the heat leaks on the performance of the Vuilleumier refrigerator, we show the $\mathrm{COP}_{\text {Leak }}$ for the standard case with $\kappa_{\text {Leak }}=5 \mathrm{~W} / \mathrm{K}$ in Figure 7 . As already pointed out above, the energy fluxes through the heat leaks are independent of the engine speed and become particularly important at low speeds. On the left side of Figure 7, this effect can be observed for high regeneration in both the OS case and the ST case, where it leads to a maximum of the $\mathrm{COP}_{\text {Leak }}$ at low speeds. On the right of Figure 7 , one sees that this effect is also present for low regeneration, but not strong enough to lead to a similarly distinct maximum of $\mathrm{COP}_{\text {Leak }}$ in the range of engine speeds considered here.

In contrast to the $\mathrm{COP}$ of the base case without heat leaks, the $\mathrm{COP}_{\text {Leak }}$ for the standard case with $\kappa_{\text {Leak }}=5 \mathrm{~W} / \mathrm{K}$ features maximum values in the considered range of engine speeds, which are reduced by about $30 \%$ for the high-regeneration OS case. Comparing the high and low regeneration cases, both the $\mathrm{COP}$ and the $\mathrm{COP}_{\text {Leak }}$ show a drastic reduction 
in efficiency for reduced regeneration. Both drop by about $50 \%$ when the regeneration coefficients are reduced by $10 \%$. This stresses once again, how important the regeneration is for the overall performance of the Vuilleumier refrigerator.
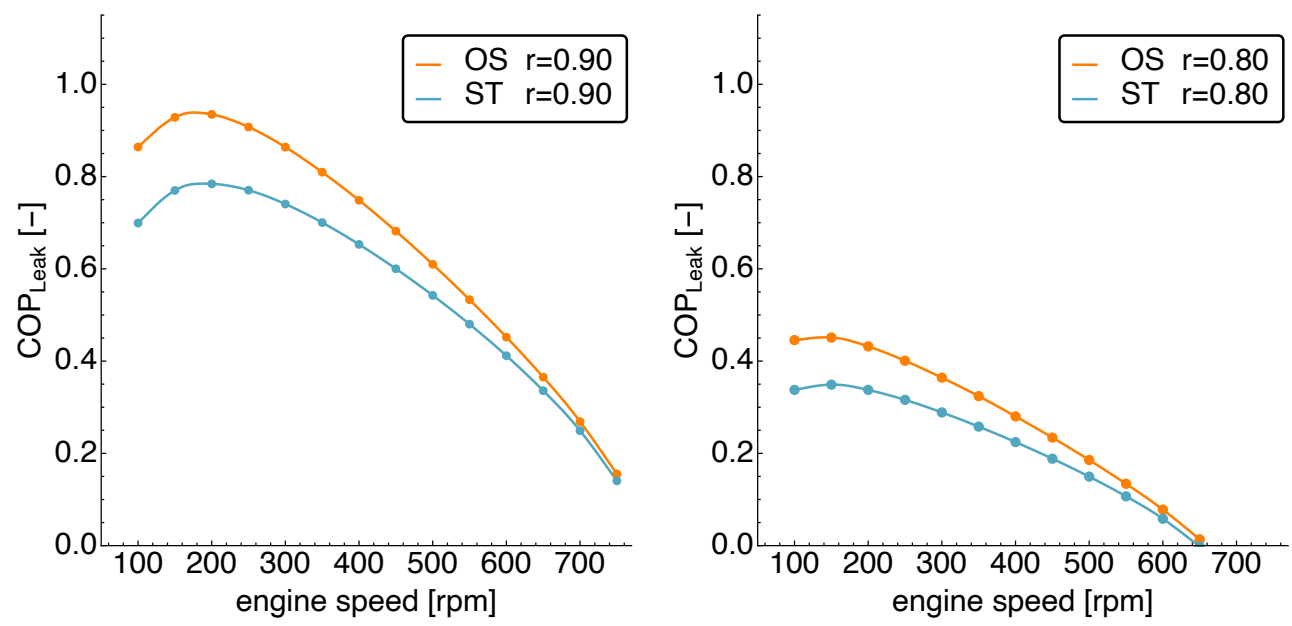

Figure 7. The standard case COP vs. engine speed for OS and ST motion at different values of the regeneration coefficient (left/right) under the influence of a heat leak of $\kappa_{\text {Leak }}=5 \mathrm{~W} / \mathrm{K}$. Again, a larger regeneration coefficient allows for higher COP values, and the slower the engine operates, the larger the COP gain by optimized piston motion (OS) is. At very low operating speeds, the standard case COP decreases due to the heat leak.

\subsection{Heat-Only Operation: Displacement Ratio}

A heat-only operation requires that the frictional losses are compensated by mechanical power obtained from the volume work of the working spaces captured by the pistons. For this purpose, the overall gas volume must change over the cycle, which typically happens in real Vuilleumier refrigerators due to the finite diameter of the driving rods of the displacers. In the particular configuration considered in our theoretical analysis, it leads to the requirement that the displacement $D_{1}$ must be larger than its counterpart $D_{31}$. In Figure 8 the ratio $D_{1} / D_{31}$ is shown as a function of the engine speed. The ratio increases with the engine speed. Interestingly, the ratio for the OS and ST cases are close together, with the OS case being larger in both regeneration cases. Note that the high regeneration case requires larger displacement ratios only at larger engine speeds.

\subsection{Optimized Piston Motion: Dynamics}

We now turn to the optimized piston motion and its dynamical features. Figures 9-12 present a comparison of the optimized sinusoidal (OS) motion for the maximum cooling power at $450 \mathrm{rpm}$ against the standard sinusoidal (ST) motion at the same speed.

The volume dynamics is shown Figure 9. On the left, the harmonic standard motion is displayed, while the optimized motion can be seen on the right. The OS motion shows, especially for $V_{1}$, a clear prolongation of the times spent close to the volume extremes, while for $V_{2}$ this is less prominent but also visible. The $V_{3}$ dynamics is-due to the chosen refrigerator configuration-given through the $V_{1}, V_{2}$ dynamics. 

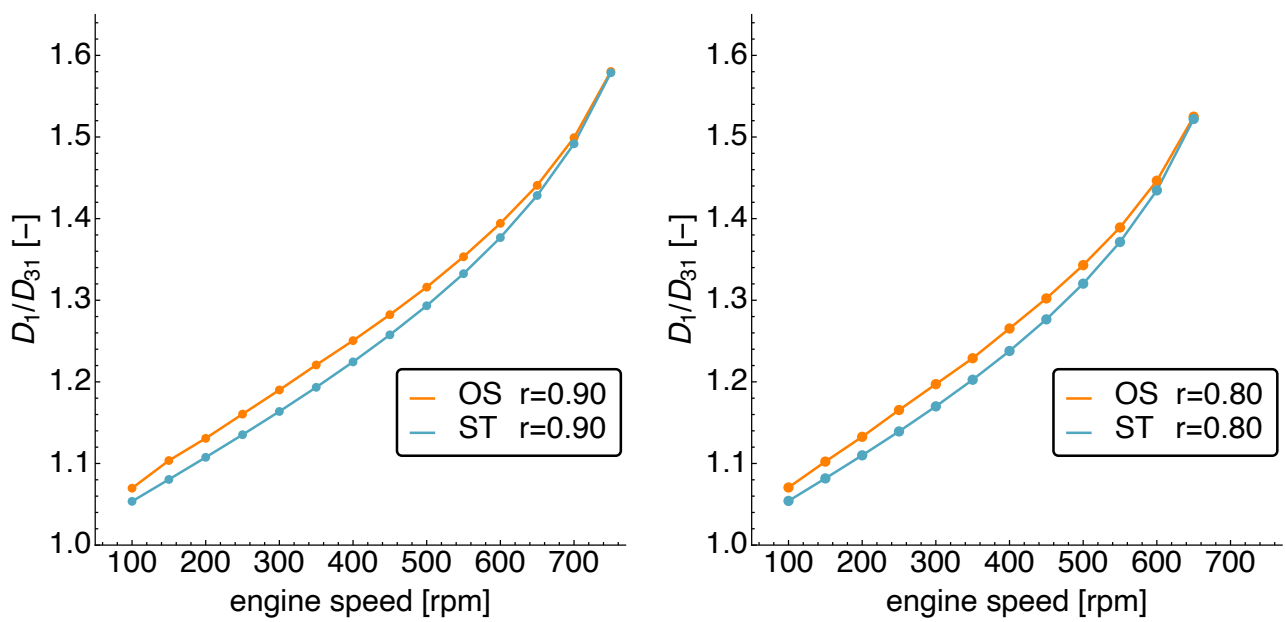

Figure 8. The displacement ratio $D_{1} / D_{31}$ necessary to gain sufficient mechanical power to overcome the friction vs. engine speed for OS and ST motion at different values of the regeneration coefficient (left/right). With increasing speed, the displacement ratio increases more than linear. For highregeneration as well as high-operation speed a slightly larger displacement ratio is necessary. Note that the optimized motion (OS) necessitates a larger displacement ratio as compared with the standard motion (ST).
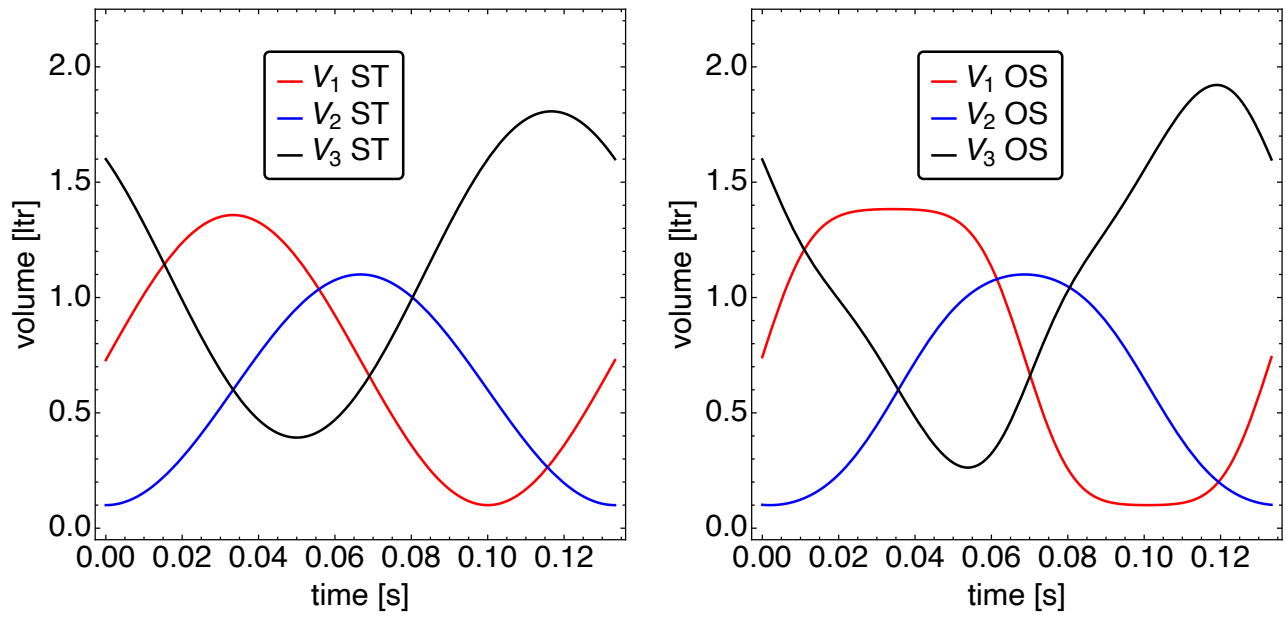

Figure 9. The volume of reservoirs 1, 2, and 3 vs. time during one cycle at $450 \mathrm{rpm}$ for ST motion (left) and OS motion (right). Comparing the respective trajectories, one sees that the trajectory of the hot regenerator shows a more square-like shape, while the trajectory of the cold regenerator is still close to a sinusoidal shape.

The effects of the altered volume dynamics also show up in the pressure and temperature dynamics.

In Figure 10 the temperature differences between the working spaces and their corresponding heat bath are shown. One notices that for the OS motion, the temperature differences are, on average, larger than for the ST motion. This is a consequence of the increased heat flows necessary for the maximized cooling power. In the ST motion, quick changes of the hot temperature around the minimum and maximum of the volume dynamics can be observed. Overall, the observed temperature differences are small compared to the absolute temperatures. 

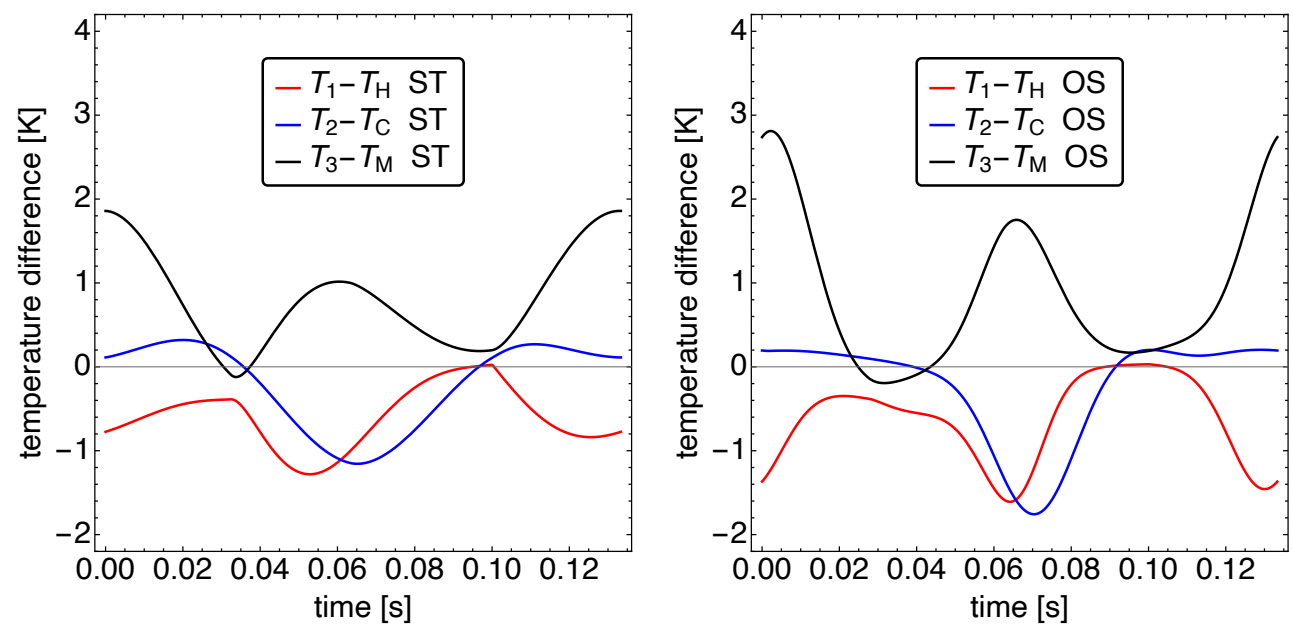

Figure 10. The temperature differences between the internal reservoirs $T_{1}, T_{2}$, and $T_{3}$ and the corresponding external reservoirs $T_{\mathrm{H}}, T_{\mathrm{C}}$, and $T_{\mathrm{M}}$ vs. time during one cycle at $450 \mathrm{rpm}$ for ST motion (left) and OS motion (right). The figure gives direct insight into the the heat flows, which are proportional to the respective temperature differences. The figure reveals that there are two periods during the cycle in which all heat flows are small, separating a phase in which the hot and cold reservoirs receive heat; the medium reservoir discharges heat from a phase in which the cold heat flux remains small while the hot reservoir gains and the medium reservoir looses heat.

In Figure 11 the pressures are shown as a function of time, while in Figure 12 the pressure differences between adjacent working spaces are displayed.

It is apparent that the pressures do not differ much between the working spaces, which is a consequence of the large mass transportation coefficient $\alpha_{0}$. For the ST motion the pressures follow a close-to-harmonic dynamics, while in the OS motion one finds stretches of almost linearly decreasing and increasing pressures. The pressures show a variation of about 10 bar around an average of 60 bar.
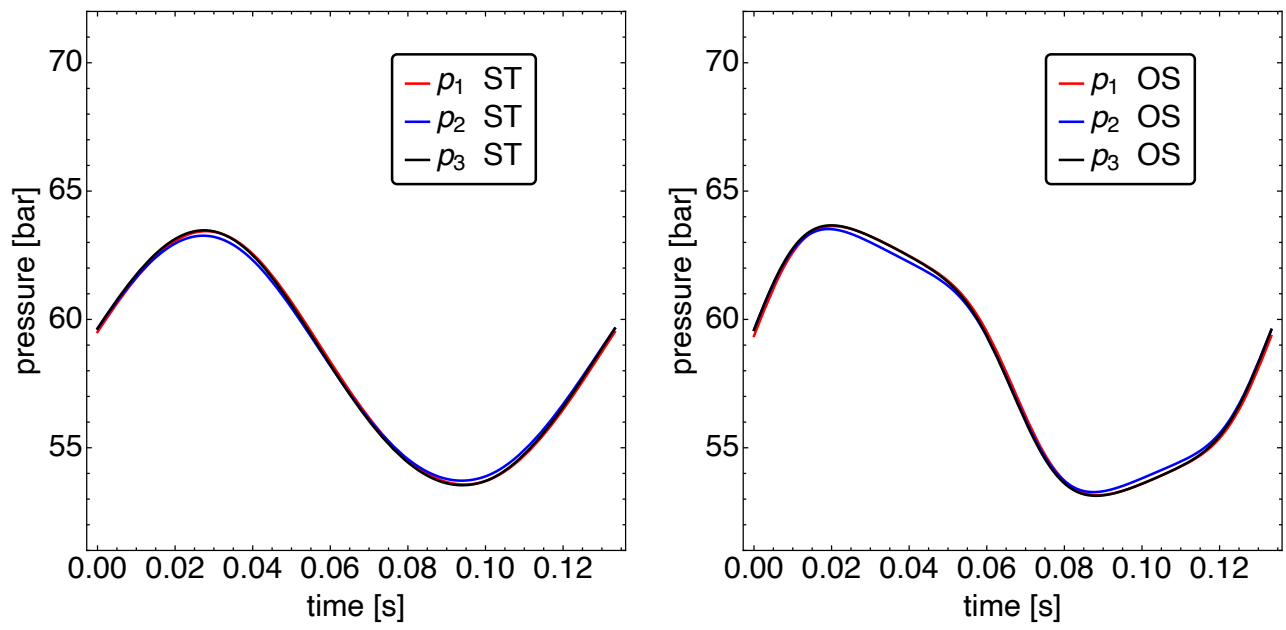

Figure 11. The pressures $p_{1}, p_{2}$, and $p_{3}$ of the three reservoirs vs. time during one cycle at $450 \mathrm{rpm}$ for ST motion (left) and OS motion (right). In general, the pressure differences between the reservoirs are small compared to the absolute pressure values. We note the distortion of the pressure trajectories for the OS motion due to the more square-like motion of the hot piston. 

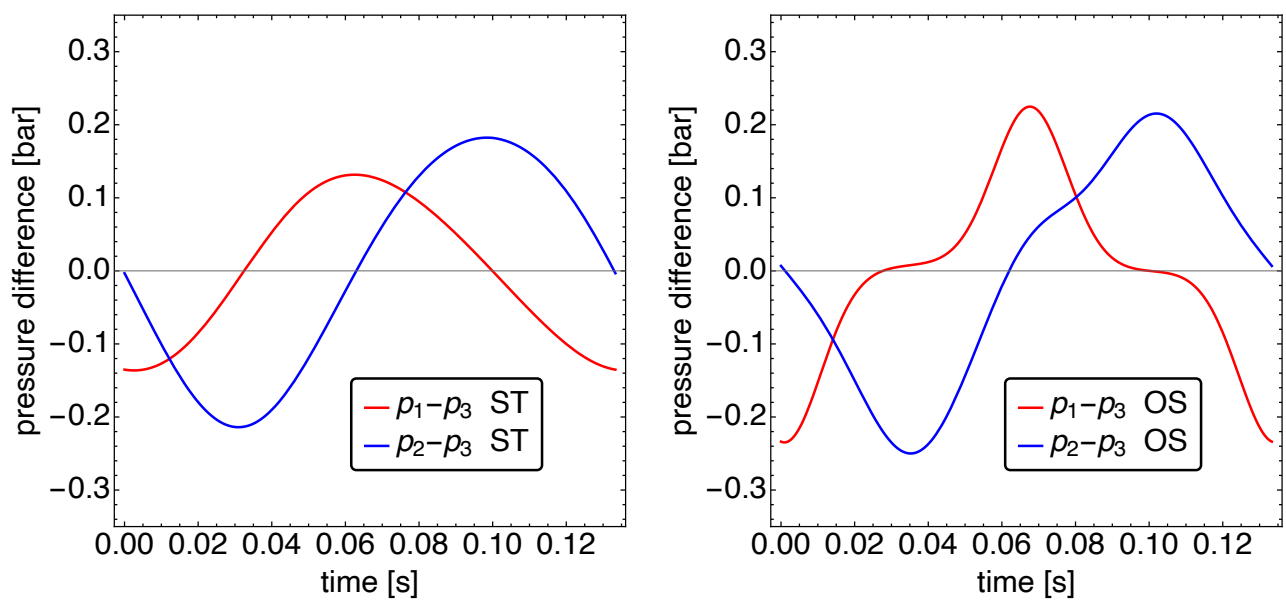

Figure 12. The pressure difference $p_{1}-p_{3}$ and $p_{2}-p_{3}$ across the hot and cold piston vs. time during one cycle at $450 \mathrm{rpm}$ for ST motion (left) and OS motion (right). These differences are caused by the gas flow resistance of the regenerators.

The pressure differences in Figure 12 reveal the important changes in the pressure dynamics. This is especially apparent in the pressure difference between working spaces 1 and 3. Here, stretches of a close-to-zero pressure difference occur in the OS motion, which have no analogue in the ST case. Note that pressure differences allow a direct view on the mass fluxes between the working spaces. The OS motion shows pronounced enlarged mass fluxes on the hot side of the refrigerator, with comparable maximal mass flux values for the hot and cold refrigerator sides.

\section{Conclusions}

The goal of this research effort was to investigate the performance features of heat-only driven Vuilleumier refrigerators. In particular, we were interested in the possible cooling power gains by optimizing the piston motion. For realistic results, we considered the main loss mechanisms (i.e., heat leakage and mechanical friction) in our endoreversible modeling as well as the effects of limited regeneration. To optimize the motion of the Vuilleumier refrigerator's regenerator pistons, we employed the adjustable sinusoidal (AS) motion class.

Our analysis showed that possible performance gains depend clearly on the extent of regeneration. We presented optimized piston motions for a high-regeneration case $(r=0.9)$ and a low-regeneration case $(r=0.8)$. In the case of high regeneration, the optimized AS motion led to a gain of about $17 \%$ in cooling power as compared to the standard sinusoidal motion, while for the low-regeneration case, the performance gain was about $28 \%$. This shows that with decreasing regeneration, the potential gain by an optimized motion increases considerably. A possible explanation for this feature is, that for lower regeneration the losses are generally larger and thus the optimization potential of an optimized piston motion is larger as well. Of course, it is always advisable to reduce mechanical losses and to increase the regeneration and heat transfer coefficients. Nonetheless, applying optimized piston motion allows for even larger gains at less favorable boundary conditions.

The importance of heat leaks for the operation of Vuilleumier refrigerators becomes apparent in the comparison of the COPs with and without the inclusion of a heat leak. Especially at lower engine speeds, a sizable decrease in COP under the influence of a heat leak of about $30 \%$ for the chosen model parameters can be observed. But we note, that in waste heat applications COP considerations are often less important than the cooling power optimization.

In future work, the application of control theory methods can show to what extent a fully optimized piston motion can increase the cooling performance even further. In addition, the use of more elaborate regenerator models will shed light on the performance 
gains possible in real refrigerators. An interesting open problem, especially for applications using waste heat, is the optimization of the auxiliary power and its minimization. The heat-only operation discussed here certainly needs further work in order to establish design rules for such refrigerators.

Author Contributions: All authors contributed equally to this article. All authors have read and agreed to the published version of the manuscript.

Funding: This research was funded by the German Federal Ministry of Education and Research under support code 01LY1706B. The publication of this article was funded by Chemnitz University of Technology.

Conflicts of Interest: The authors declare no conflicts of interest.

\section{References}

1. Vuilleumier, R. Method and Apparatus for Inducing Heat Changes. U.S. Patent 1,275,507, 13 August 1918.

2. Dogkas, G.; Rogdakis, E. A review on Vuilleumier machines. Therm. Sc. Eng. Prog. 2018, 8, 340-354. [CrossRef]

3. Carlsen, H. Development of a gas fired Vuilleumier heat pump for residential heating. In Proceedings of the 24th Intersociety Energy Conversion Engineering Conference, Washington, DC, USA, 6-11 August 1989; pp. 2257-2263. [CrossRef]

4. Chen, H.; Hofbauer, P.; Longtin, J.P. Multi-objective optimization of a free-piston Vuilleumier heat pump using a genetic algorithm. Appl. Therm. Eng. Des. Process. Equipment. Econ. 2020, 167, 114767. [CrossRef]

5. Shi, P.; Wang, L.S.; Schwartz, P.; Hofbauer, P. State-wide comparative analysis of the cost saving potential of Vuilleumier heat pumps in residential houses. Appl. Energy 2020, 277, 115547. [CrossRef]

6. Miller, W.S.; Potter, V.L. Fractional Watt Vuilleumier Cryogenic Refrigerator; Technical Report; National Aeronautics and Space Administlation: Torrance, CA, USA, 1973.

7. White, R. Vuilleumier Cycle Cryogenic Refrigeration; Technical Report; Air Force Flight Dynamics Laboratory: Wright-Patterson AFB, OH, USA, 1976.

8. Matsubara, Y.; Kaneko, M. Vuilleumier cycle cryocooler operating below 8 K. In Proceedings of the Third Cryocooler Conference, Boulder, CO, USA, 17-18 September 1984; pp. 234-239.

9. Kawada, M.; Kudo, I.; Yoshimura, H. Small Vuilleumier Cryocooler: Comparison of Performance Test Results and Calculation. Trans. Jpn. Soc. Mech. Eng. B 1995, 61, 713-721. [CrossRef]

10. Tong, Z.; Changzhao, P.; Liubiao, G.; Wenxui, Z.; Yuan, Z.; Junjie, W. Experimental study on a one-stage Vuilleumier cryocooler with large pressure ratio. IOP Conf. Ser. Mater. Sci. Eng. 2017, 171, 012077. [CrossRef]

11. Schulz, S.; Thomas, B. Experimental investigation of a free-piston Vuilleumier refrigemtor. Int. J. Refrig. 1995, 18, 51-57. [CrossRef]

12. Chen, H.; Lin, C.; Longtin, J.P. Performance analysis of a free-piston Vuilleumier heat pump with dwell-based motion. Appl. Therm. Eng. Design. Processes. Equipment. Econ. 2018, 140, 553-563. [CrossRef]

13. Chen, H.; Lin, C.; Longtin, J.P. Dynamic modeling and parameter optimization of a free-piston Vuilleumier heat pump with dwell-based motion. Appl. Energy 2019, 242, 741-751. [CrossRef]

14. Hofbauer, P. Four-Process Cycle for a Vuilleumier Heat Pump. U.S. Patent 10,030,893, 13 October 2018.

15. Paul, R.; Hoffmann, K.H. Cyclic Control Optimization Algorithm for Stirling Engines. Symmetry 2021, 13, 873. [CrossRef]

16. Craun, M.; Bamieh, B. Optimal Periodic Control of an Ideal Stirling Engine Model. J. Dyn. Syst. Meas. Control 2015, 137, 071002. [CrossRef]

17. Masser, R.; Khodja, A.; Scheunert, M.; Schwalbe, K.; Fischer, A.; Paul, R.; Hoffmann, K.H. Optimized Piston Motion for an Alpha-Type Stirling Engine. Entropy 2020, 22, 700. [CrossRef]

18. Scheunert, M.; Masser, R.; Khodja, A.; Paul, R.; Schwalbe, K.; Fischer, A.; Hoffmann, K.H. Power-Optimized Sinusoidal Piston Motion and Its Performance Gain for an Alpha-Type Stirling Engine with Limited Regeneration. Energies 2020, 13, 4564. [CrossRef]

19. Erdman, P.A.; Cavina, V.; Fazio, R.; Taddei, F.; Giovannetti, V. Maximum power and corresponding efficiency for two-level heat engines and refrigerators: Optimality of fast cycles. New J. Phys. 2019, 21, 103049. [CrossRef]

20. Hoffmann, K.H.; Watowich, S.J.; Berry, R.S. Optimal Paths for Thermodynamic Systems: The Ideal Diesel Cycle. J. Appl. Phys. 1985, 58, 2125-2134. [CrossRef]

21. Burzler, J.M.; Blaudeck, P.; Hoffmann, K.H. Optimal Piston Paths for Diesel Engines. In Thermodynamics of Energy Conversion and Transport; Stanislaw Sieniutycz, S., de Vos, A., Eds.; Springer: Berlin/Heidelberg, Germany, 2000; pp. 173-198. [CrossRef]

22. Chen, L.; Xia, S.; Sun, F. Optimizing piston velocity profile for maximum work output from a generalized radiative law Diesel engine. Math. Comput. Model. 2011, 54, 2051-2063. [CrossRef]

23. Watowich, S.J.; Hoffmann, K.H.; Berry, R.S. Intrinsically Irreversible Light-Driven Engine. J. Appl. Phys. 1985, 58, $2893-2901$. [CrossRef]

24. Watowich, S.J.; Hoffmann, K.H.; Berry, R.S. Optimal Paths for a Bimolecular, Light-Driven Engine. Il Nuovo Cimento B 1989, 104, 131-147. [CrossRef] 
25. Ma, K.; Chen, L.; Sun, F. Optimal paths for a light-driven engine with a linear phenomenological heat transfer law. Sci. China. Chem. 2010, 53, 917-926. [CrossRef]

26. Hoffmann, K.H.; Burzler, J.M.; Schubert, S. Endoreversible Thermodynamics. J. Non-Equilib. Thermodyn. 1997, 22, $311-355$.

27. Hoffmann, K.H.; Burzler, J.M.; Fischer, A.; Schaller, M.; Schubert, S. Optimal Process Paths for Endoreversible Systems. J. Non-Equilib. Thermodyn. 2003, 28, 233-268. [CrossRef]

28. Hoffmann, K.H. An introduction to endoreversible thermodynamics. Messanae Univ. Stud. 2008, 86, 1-19. [CrossRef]

29. Shang, Y. Fixed-time group consensus for multi-agent systems with non-linear dynamics and uncertainties. IET Control Theory Appl. 2018, 12, 395-404. [CrossRef]

30. Bădescu, V. The Theoretical Maximum Efficiency of Solar Converters with and Without Concentration. Energy 1989, 14, 237-239. [CrossRef]

31. De Vos, A. Is a solar cell an endoreversible engine? Sol. Cells 1991, 31, 181-196. [CrossRef]

32. Schwalbe, K.; Hoffmann, K.H. Optimal Control of an Endoreversible Solar Power Plant. J. Non-Equilib. Thermodyn. 2018, 43, 255-271. [CrossRef]

33. Schwalbe, K.; Hoffmann, K.H. Stochastic Novikov engine with time dependent temperature fluctuations. Appl. Therm. Eng. Design. Processes. Equipment. Econ. 2018, 142, 483-488. [CrossRef]

34. Wagner, K.; Hoffmann, K.H. Endoreversible modeling of a PEM fuel cell. J. Non-Equilib. Thermodyn. 2015, 40, 283-294. [CrossRef]

35. Gordon, J.M.; Orlov, V.N. Performance Characteristics of Endoreversible Chemical Engines. J. Appl. Phys. 1993, 74, 5303-5309. [CrossRef]

36. Xia, S.; Chen, L.; Sun, F. Maximum power configuration for multireservoir chemical engines. J. Appl. Phys. 2009, 105, 1-6. [CrossRef]

37. Wagner, K.; Hoffmann, K.H. Chemical reactions in endoreversible thermodynamics. Eur. J. Phys. 2016, 37, 015101. [CrossRef]

38. Marsik, F.; Weigand, B.; Thomas, M.; Tucek, O.; Novotny, P. On the Efficiency of Electrochemical Devices from the Perspective of Endoreversible Thermodynamics. J. Non-Equilib. Thermodyn. 2019, 44, 425-437. [CrossRef]

39. Muschik, W.; Hoffmann, K. Endoreversible Thermodynamics: A Tool for Simulating and Comparing Processes of Discrete Systems. JNET 2006, 31, 293-317. [CrossRef]

40. Gonzalez-Ayala, J.; Mateos Roco, J.M.; Medina, A.; Calvo Hernández, A. Optimization, Stability, and Entropy in Endoreversible Heat Engines. Entropy 2020, 22, 1323. [CrossRef] [PubMed]

41. Muschik, W.; Hoffmann, K.H. Modeling, Simulation, and Reconstruction of 2-Reservoir Heat-to-Power Processes in Finite-Time Thermodynamics. Entropy 2020, 22, 997. [CrossRef] [PubMed]

42. Smith, Z.; Pal, P.S.; Deffner, S. Endoreversible Otto Engines at Maximal Power. J. Non-Equilib. Thermodyn. 2020, 45, 305-310. [CrossRef]

43. Schwalbe, K.; Hoffmann, K.H. Novikov engine with fluctuating heat bath temperature. J. Non-Equilib. Thermodyn. 2018, 43, 141-150. [CrossRef]

44. Schwalbe, K.; Hoffmann, K.H. Performance Features of a Stationary Stochastic Novikov Engine. Entropy 2018, 20, 52. [CrossRef]

45. Schwalbe, K.; Hoffmann, K.H. Stochastic Novikov Engine with Fourier Heat Transport. J. Non-Equilib. Thermodyn. 2019, 44, 417-424. [CrossRef]

46. Barranco-Jiménez, M.A.; Ocampo-García, A.; Angulo-Brown, F. Thermodynamic analysis of an array of isothermal endoreversible electric engines. Eur. Phys. J. Plus 2020, 135, 153. [CrossRef]

47. Chen, L.; Meng, F.; Ge, Y.; Feng, H. Performance Optimization for a Multielement Thermoelectric Refrigerator with Linear Phenomenological Heat Transfer Law. J. Non-Equilib. Thermodyn. 2021, 46, 149-162:10.1515/jnet-2020-0050. [CrossRef]

48. Feidt, M.; Costea, M.; Feidt, R.; Danel, Q.; Périlhon, C. New Criteria to Characterize the Waste Heat Recovery. Energies 2020, 13, 789. [CrossRef]

49. Levario-Medina, S.; Valencia-Ortega, G.; Barranco-Jiménez, M.A. Energetic Optimization Considering a Generalization of the Ecological Criterion in TraditionalSimple-Cycle and Combined-Cycle Power Plants. JNET 2020, 45, 269-290. [CrossRef]

50. Masser, R.; Hoffmann, K.H. Optimal Control for a Hydraulic Recuperation System Using Endoreversible Thermodynamics. Appl. Sci. 2021, 11, 5001. [CrossRef]

51. Wu, Z.; Chen, L.; Feng, H. Thermodynamic Optimization for an Endoreversible Dual-Miller Cycle (DMC) with Finite Speed of Piston. Entropy 2018, 20, 165. [CrossRef] [PubMed]

52. Meng, Z.; Chen, L.; Wu, F. Optimal Power and Efficiency of Multi-Stage Endoreversible Quantum Carnot Heat Engine with Harmonic Oscillators at the Classical Limit. Entropy 2020, 22, 457. [CrossRef] [PubMed]

53. Essex, C.; Andresen, B. The principal equation of state for classical particles, photons, and neutrinos. J. Non-Equilib. Thermodyn. 2013, 38, 293-312. [CrossRef]

54. Kühl, H.D.; Schulz, S. A 2nd order regenerator model including flow dispersion and bypass losses. In Proceedings of the 31st Intersociety Energy Conversion Engineering Conference (IECEC 96), Washington, DC, USA, 11-16 August 1996; Volume 2, pp. 1343-1348. [CrossRef]

55. Paul, R.; Hoffmann, K.H. A Class of Reduced-Order Regenerator Models. Energies 2021, 14, 7295. [CrossRef]

56. Paul, R.; Khodja, A.; Hoffmann, K.H. An Endoreversible Model for the Regenerators of Vuilleumier Refrigerators. Int. J. Thermodyn. 2021, 24, 184-192. [CrossRef] 
57. Moukalled, F.; Nuwayhid, R.Y.; Noueihed, N. The Efficiency of Endoreversible Heat Engines with Heat Leak. Int. J. Energy Res. 1995, 19, 377-389. [CrossRef]

58. Huleihil, M.; Andresen, B. Effects of heat leak on the performance characteristics of Carnot like heat engines and heat pumps. Lat.-Am. J. Phys. Educ. 2011, 5, 16-21.

59. Nelder, J.A.; Mead, R. A Simplex Method for Function Minimization. Comput. J. 1965, 7, 308-313. [CrossRef] 